livraisons

d'Histoire

de l'Architecture

\section{Livraisons de l'histoire de l'architecture}

14 | 2007

Piscines

\title{
Strasbourg, Colmar, Mulhouse : les programmes de bains municipaux en Alsace au début du XXe siècle. Une politique volontaire d'hygiène publique
}

Strasbourg, Colmar, Mulhouse: the municipal baths programs in Alsace in the early $X X^{\text {th }}$ century, about a wilful policy on public health Straßburg, Colmar, Mülhausen : Bauprogramme von städtischen Bädern im Elsass anfangs des 20.Jahrhunderts, eine resolute Politik für öffentliche Hygiene

Patrice Triboux

\section{OpenEdition}

\section{Journals}

Édition électronique

URL : http://journals.openedition.org/lha/430

DOI : $10.4000 /$ /ha. 430

ISSN : 1960-5994

Éditeur

Association Livraisons d'histoire de l'architecture - LHA

Édition imprimée

Date de publication : 10 décembre 2007

Pagination : 81-93

ISSN : 1627-4970

Référence électronique

Patrice Triboux, «Strasbourg, Colmar, Mulhouse : les programmes de bains municipaux en Alsace au début du XXe siècle. Une politique volontaire d'hygiène publique », Livraisons de l'histoire de

l'architecture [En ligne], 14 | 2007, mis en ligne le 10 décembre 2009, consulté le 19 avril 2019. URL: http://journals.openedition.org//ha/430 ; DOl : 10.4000//ha.430

Ce document a été généré automatiquement le 19 avril 2019

Tous droits réservés à l'Association LHA 


\title{
Strasbourg, Colmar, Mulhouse : les programmes de bains municipaux en Alsace au début du XXe siècle. Une politique volontaire d'hygiène publique
}

\author{
Strasbourg, Colmar, Mulhouse: the municipal baths programs in Alsace in the \\ early $X X^{\text {th }}$ century, about a wilful policy on public health \\ Straßburg, Colmar, Mülhausen : Bauprogramme von städtischen Bädern im \\ Elsass anfangs des 20.Jahrhunderts, eine resolute Politik für öffentliche Hygiene
}

\section{Patrice Triboux}

Dans ses rapports architecturaux avec l'eau, l'Alsace entretient une relation privilégiée puisque la tradition des bains y est établie de longue date et connaît, à la fin du Moyen Âge, une période propice où l'on trouve, ouverts au public, étuves, bains en cuve et bains aromatiques ${ }^{1}$. Aucune cité d'importance ne s'est abstenue de s'en doter ${ }^{2}$. Au cours du XIX siècle, à Strasbourg comme à Mulhouse et Colmar, les bains de rivière et les bains publics organisés et gérés par les municipalités ont précédé la construction d'un programme intégré de bains municipaux avec bassins de natation, rendus désormais nécessaires par le fort accroissement de la population urbaine. L'âge des piscines à vocation sportive commence au début du XXe siècle, alors que l'Alsace est annexée à l'Allemagne. Cette période d'intense modification et d'extension urbaine de Strasbourg, Colmar et Mulhouse, voit se concrétiser trois importants programmes de bains municipaux conjuguant hygiène et loisir, bassins de natation et bains médicaux dans l'esprit des programmes déterminés en Europe centrale et en Allemagne à la charnière des $\mathrm{XIX}^{\mathrm{e}}$ et $\mathrm{XX}^{\mathrm{e}}$ siècles.

Les bains municipaux de Strasbourg, en projet dès 1898, sont inaugurés en 1908. Ils sont les plus ambitieux et liés à la volonté municipale de contrer l'administration impériale en matière de transformations architecturales de la ville. Ceux de Colmar sont approuvés par 
le conseil municipal du 28 septembre 1904 et ouvrent leurs portes en 1906. Enfin, ceux de Mulhouse, conçus à partir de 1911 ne sont inaugurés qu'en 1925. Ce sont, au début du XX ${ }^{\mathrm{e}}$ siècle, les trois piscines les plus modernes d'Alsace, qui reflètent la volonté des édiles locaux de fournir à la population des équipements intégrés offrant à la fois une dimension sportive, sanitaire et sociale. Le cas de la piscine de Guebwiller (détruite) ne sera pas évoqué ici bien qu'elle fût la première des piscines construites en Alsace en béton armé par l'entrepreneur Édouard Zublin, entre 1898 et 1900.

\section{Piscines et béton armé}

3 La maison Zublin \& C $\mathrm{C}^{\mathrm{ie}}$, alors installée à Strasbourg, est, en effet, chargée du gros œuvre des trois établissements. L'importance de cette compagnie, pionnière dans l'expérimentation et la mise en œuvre du béton armé, fait de ces piscines les réalisations les plus novatrices sur le plan technique. Outre le Palais des Fêtes conçu par les architectes Kuder et Müller en 1903, les bains municipaux figurent parmi les premières applications du béton armé à Strasbourg. Le catalogue des réalisations menées par Zublin publié en 1923 consacre dans sa section «Architecture» un encart consacré aux bains publics. On peut y lire :

Les questions d'hygiène et de culture physique ont pris, depuis une vingtaine d'années, une grande importance et de remarquables progrès ont été accomplis dans les institutions qui s'occupent du développement du corps humain. C'est pourquoi nous consacrons un chapitre aux établissements de bains populaires dont un certain nombre, entre autres, ceux de Colmar, Guebwiller, Markirch et Strasbourg ont été construits par la maison Ed. Zublin et $\mathrm{C}^{\text {ie }}$.

5 La nouveauté consiste en effet à utiliser le béton armé au lieu du béton damé, non seulement pour la réalisation des bassins, mais aussi pour les planchers et la couverture. L'étanchéité nouvellement obtenue par les propriétés physiques du béton armé dispensait ainsi « de forts revêtements et garnitures » ${ }^{4}$

6 Le bureau d'études de Zublin met en œuvre le béton armé pour la voûte en berceau à lunette et, au niveau du bassin, réalise une coque de béton armé reposant sur des pieux de fondation, telle que le montre la coupe transversale sur le bassin des hommes réalisé à Mulhouse ${ }^{5}$. La maison Zublin précise par ailleurs que, pour en permettre la dilatation, le bassin n'est fixé sur le sol qu'à une extrémité, les autres parties étant supportées par des piliers et séparées des planchers par des joints de dilatation de trois centimètres, comme cela se vérifie sur la coupe longitudinale et le plan du bassin des hommes de Mulhouse ${ }^{6}$. La stabilité de l'ensemble étant ainsi assurée, un simple enduit recouvre la cavité afin d'en assurer l'étanchéité absolue, avant d'être revêtu de catelles blanches. Enfin, la protection du bassin et son isolation exigent l'emploi de parois et de planchers isolants creux et de toitures à double cloison ${ }^{7}$. La même technique est scrupuleusement utilisée à Strasbourg, à Mulhouse et à Colmar. Après la première guerre mondiale, Zublin acquiert une excellente réputation dans le domaine de la construction en béton armé et intervient tant en France (grands moulins de Pantin, ouvrages d'art...) qu'en Allemagne. 


\section{Les piscines de Strasbourg}

$7 \quad$ L'idée de doter la ville de Strasbourg de bains municipaux date de la séance du conseil municipal du 30 juin 1894 présidée par le maire Otto Back, mais la décision de poursuivre le projet ne sera prise qu'en octobre 1898. Johann Karl Ott, architecte de la ville, présente un premier projet en 1901. Ce n'est qu'avec l'arrivée à Strasbourg de l'architecte Fritz Beblo au poste de Stadtbauinspecktor auprès du service municipal d'architecture, que le projet aboutit. Fritz Beblo précise néanmoins s'être inspiré de la conception d'ensemble pensée par son prédécesseur, le Stadtbaurat Karl Ott. Beblo est assisté sur ce chantier par l'architecte Ernst Fettig, un de ses collaborateurs habituels, qui signe de nombreux plans. En 1905, année de l'inauguration du Palais des Fêtes à Strasbourg, débutent les travaux du gros œuvre. L'année suivante, la commission municipale visite des piscines en Allemagne et notamment Augsbourg, Düsseldorf, Francfort, Giessen, Munich, Stuttgart, afin d'évaluer et de comparer les réalisations les unes aux autres. À la suite de cette visite, des modifications interviennent sur le projet initial. Après plusieurs projets concernant le système de charpente, les travaux s'achèvent et l'établissement de bains municipaux de Strasbourg est inauguré en août 1908 avec les deux bassins de natation; le bâtiment annexe des bains médicinaux n'étant quant à lui opérationnel qu'en 1911. Des transformations ultérieures sont entreprises jusqu'en $1942^{8}$.

8 Le coût final du projet s'élève à 1500000 marks ce qui en fait le projet le plus coûteux mené par Beblo durant son activité à Strasbourg. Les bains municipaux constituent l'une des œuvres les plus abouties de l'architecte', particulièrement actif comme Stadtbauinspektor puis Stadtbaurat entre 1903 et $1918^{10}$ dont le style est proche des maîtres de Darmstadt et de Karlsruhe, Josef Olbrich et Hermann Billing. En comparaison, les réalisations de son prédécesseur, le Stadtbaurat Ott, offrent un style néo-renaissance germanique alors que les bains de Beblo parviennent à allier nouveauté technique (emploi du béton armé) et éclectisme architectural teinté de régionalisme pittoresque non dépourvu de légers accents Jugendstil. Il « rappelle la tradition par sa manière de faire la cohésion entre les habitudes optiques et les modernismes tactiles ${ }^{11} »$.

9 Pour le décor, l'architecte fait appel à différentes entreprises allemandes dont certaines strasbourgeoises comme la maison Ott Frères, chargée de la réalisation des cartons des vitraux ou le peintre verrier Joseph Ehrismann (1880-1937) qui signe les verrières des deux oculi des bains romains. La Steinindustrie Schachenmühle fournit les marbres et les pierres dures ; le décor peint et les stucs sont réalisés par Maechling \& Cie, la peinture et les enduits par la Firma Griesbach und Grossmann de Strasbourg tandis que les travaux de serrurerie d'art sont l'œuvre de Haug, Hagenstein, Unselt, Roehlend \& Brennstuhl, Rupp.

Le nouvel établissement des bains est implanté au sud du Palais universitaire et de l'axe est-ouest qu'il forme avec le Palais impérial ${ }^{12}$, sur le Nikolausring, actuel boulevard de la Victoire, non loin de la caserne de la Porte des Pêcheurs, en limite du quartier dit "allemand » ${ }^{13}$. La parcelle triangulaire a nécessairement posé des contraintes à l'implantation de deux bassins rectangulaires, mais prouve la volonté municipale de faire des bains un équipement de proximité pour la population, ce qui en justifie le coût élevé, tout en parvenant à l'ériger en vitrine fastueuse pour les nouvelles piscines. Le plan de $1906^{14}$ montre clairement la juxtaposition des volumes dévolus chacun à une activité différente : deux masses disposées orthogonalement abritent les bassins de natation de part et d'autre d'un espace intermédiaire consacré aux bains médicinaux, le tout situé 
derrière la façade antérieure abritant les bains en baignoire et le grand vestibule de réception ovale saillant en façade ${ }^{15}$. Cette tripartition de l'espace à partir de la rotonde de réception de l'établissement n'impose pas de hiérarchie entre les activités : baignade, thermalisme, ou hygiène du corps. La rotonde couverte d'une coupole à lanternon comprend un niveau de galerie à portique. Le sol est revêtu d'un dallage de marbres en deux tons irradiant d'un octogone inscrit dans un disque central. Au rez-de-chaussée, des arcs en anse de panier ornés d'une agrafe stuquée à thème zoologique ou aquatique reposent sur des piles de section rectangulaire. L'agrafe axiale est ornée d'un décor de grotesques avec concrétions. À l'étage, des colonnettes en pierre dure veinées de gris rythment l'élévation et font office de tambour à la coupole nervurée de bandes décoratives. Le plafond de la galerie supérieure est scandé de huit cartouches décoratifs de style néo-rocaille disposés aux emplacements des luminaires. Les tailloirs des piles rectangulaires sont prolongés en élévation par des pilastres revêtus d'un enduit de fines stries verticales ondulées imitant des chutes d'eau. Ainsi la domestication de l'eau et l'affirmation des plaisirs qu'elle procure sont présentées dans le décor de la rotonde à dominante de couleurs tendres, sourdes et pastels, comme dans les piscines où le programme iconographique se décline sur des thèmes aquatiques, sans référence directe à la mythologie.

Les élévations des deux bassins de natation, à l'est comme à l'ouest sont clairement identifiables de l'extérieur (ill. 1).

\section{1 : Städt schwimmbad Strassburg, facade am Nicolausring}

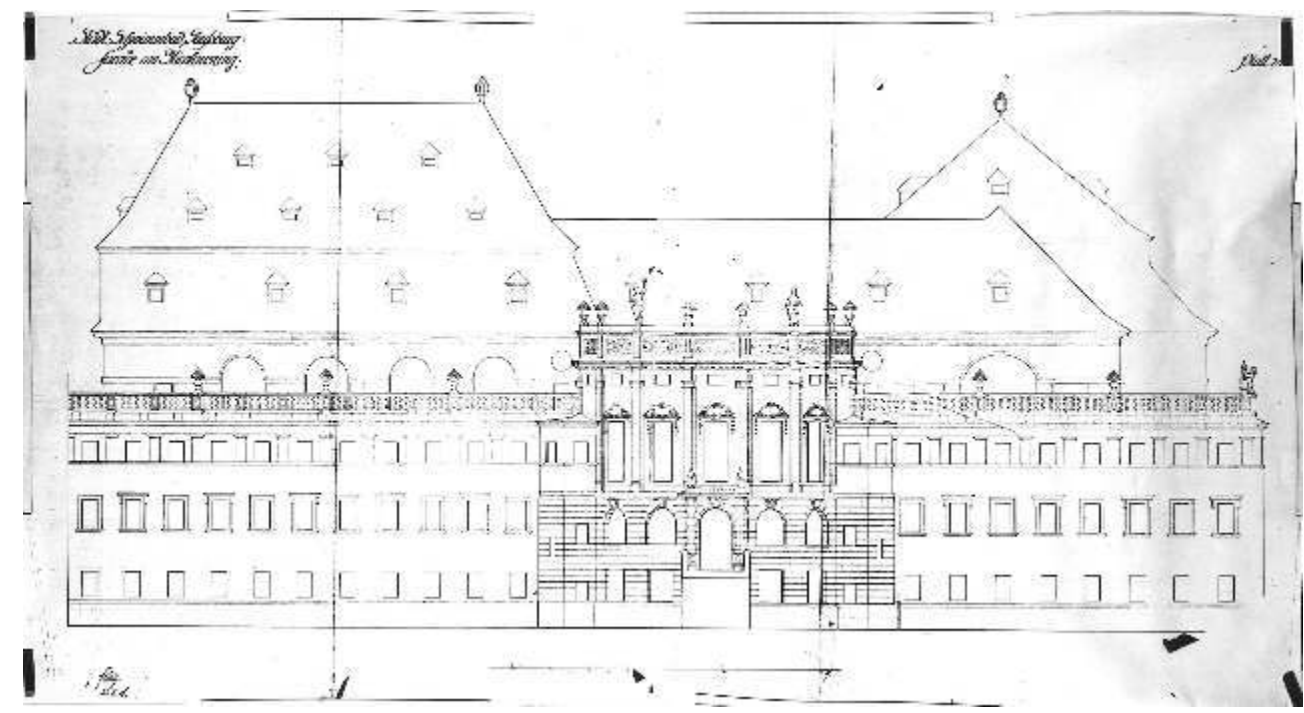

Élévation de l'aile principale nord, signée et datée : fettig $22 \times 06$

Archives municipales de Strasbourg, police du bâtiment. Cl. CRMH / B 2169.

Ils se présentent en retrait de la façade antérieure qu'ils surpassent d'un niveau d'arcades en plein cintre, de combles brisés et d'un toit à croupes et à longs pans, percé de lucarnes. Le décor et l'organisation spatiale annoncés dès la rotonde de réception se retrouvent dans les deux bâtiments hors œuvre des piscines. La voûte en béton armé en berceau à lunettes du bassin des hommes s'étend sur cinq travées, chacune d'entre elles étant ouverte, vers l'ouest, par une grande baie en plein cintre. L'intrados des lunettes est revêtu d'un décor de faux caissons octogonaux tandis que l'espace couvert par le berceau est souligné d'une corniche décorée d'agrafes ornées d'un décor végétal pour chaque 
travée. Le milieu de la voûte est percé d'oculi à grille derrière lesquels est disposé l'éclairage électrique.

L'extrémité sud du bâtiment est ouverte au rez-de-chaussée de trois baies rectangulaires et d'un vaste triplet au premier niveau qui constitue une variation sur le thème de la fenêtre thermale et une des rares références du bâtiment à l'architecture des thermes antiques. Celle-ci est ornée d'un décor de colonnes engagées avec entablement baroque sur lequel deux groupes latéraux figurent des putti déversant des jarres à côté d'une fontaine à candélabre et à vasques. La fenêtre est flanquée de volutes latérales retombant en queue de sirène. Les trois lunettes aveugles du côté oriental sont décorées d'un médaillon figurant un drakkar, un cygne de profil et un dauphin.

L'accès à l'intérieur des piscines s'effectue à l'ouest pour les dames et à l'est pour les hommes. Les deux bassins adoptent la même organisation spatiale. Un vestibule, muni d'une fontaine lavabo en marbre et revêtu au sol d'un carrelage décoratif et, paradoxalement, de forme ovale pour le bassin masculin, rectangulaire aux angles abattus pour le bassin féminin, mène aux galeries de cabines de déshabillage. Les galeries de cabines étant disposées au rez-de-chaussée et au premier étage, en arrière des bassins pour les enfants et, de part et d'autre des bassins, pour les adultes sur les deux niveaux. Le bassin de natation rectangulaire d'une dimension de $12 \mathrm{~m}$. de large sur $25 \mathrm{~m}$. de long et d'une profondeur maximale de $3 \mathrm{~m}$. est fermé, du côté sud, par une imposante fontaine de marbre beige à plot central rectangulaire, orné d'un masque à grotesque dont la bouche béante laisse jaillir l'eau. De part et d'autre de la fontaine, sur le muret séparant le bassin de l'espace des douches et des pédiluves, six niches, décorées de coquilles baroques revêtues de mosaïque d'or crachent des jets secondaires. Le bassin de natation pour dames (ill. 2), de forme cintrée à l'ouest, est plus petit: 9,5 m. de large sur $17 \mathrm{~m}$. de long pour une profondeur maximale de $2,80 \mathrm{~m}$. 
III. 2 : Anonyme, Bains municipaux de Strasbourg : vue d'ensemble du bassin de natation pour dames vers l'Ouest

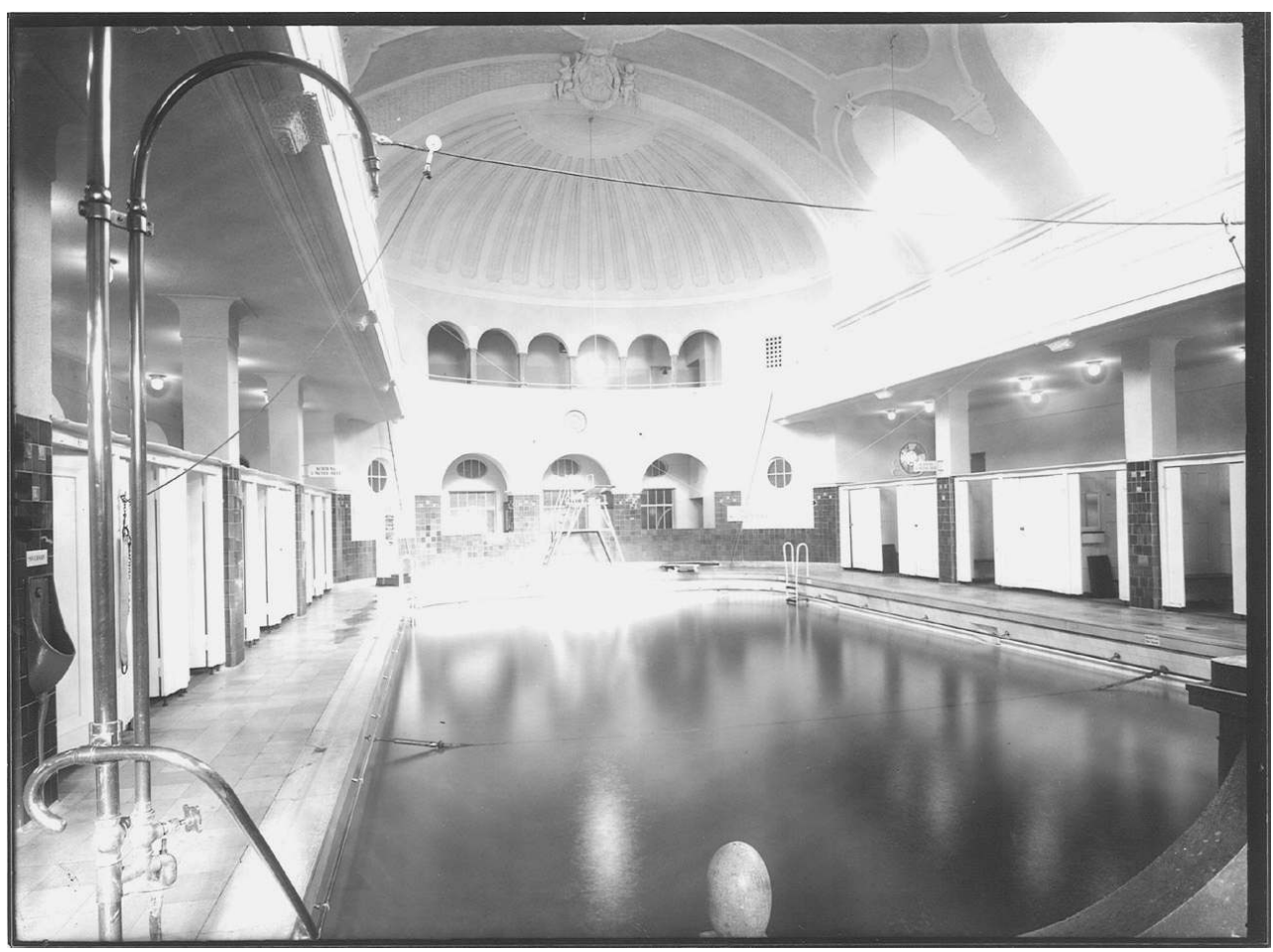

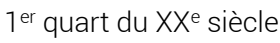

Archives des bains municipaux, Strasbourg

Couvert d'une voûte en berceau à lunettes revêtue d'un décor néo-baroque, il comporte deux niveaux de cabines superposées. Une abside en cul-de-four constituée de deux niveaux d'arcatures ouvertes sur des galeries fait office de pignon occidental. Une horloge murale à décor polychrome est insérée entre les deux niveaux. Le rang d'arcades supérieures est flanqué de deux discrets claustra, tandis que la voûte est nervurée. Au sommet de l'arc diaphragme, deux putti tiennent une guirlande encadrant un blason baroque à décor végétal. Le pignon opposé, plus sage, superpose une tribune à l'espace des douches au fond duquel s'ouvre la cage de l'escalier à vis desservant le niveau supérieur.

Les bains municipaux de Strasbourg n'ont pas pour seule vocation d'offrir un équipement sportif à la population, même si, dès 1910, leur administration établit les statistiques des fréquentations scolaires ${ }^{16}$ qui totalisent 568149 entrées entre 1910 et 1919, sans pour autant organiser un enseignement de la natation, qui ne débute que vers 1920 . L'institutionnalisation progressive au sein de l'école d'un enseignement de la natation permet aux bains municipaux d'augmenter radicalement le nombre d'écoliers nageurs et de brevets décernés, si bien que Strasbourg obtient en 1934 un prix d'honneur et les éloges du ministre de la santé publique et de l'éducation physique ${ }^{17}$. La fréquentation scolaire constitue par ailleurs un enjeu économique pour l'établissement. Il s'agit donc de doter la ville d'un équipement moderne, intégré, comprenant bains douches à vocation hygiéniste, bains médicinaux et piscines de natation, à l'instar des grandes villes allemandes qui à la veille de la première guerre mondiale, possèdent toutes ce type de structure. 
17 La construction des bains municipaux de Strasbourg sous la direction de Beblo s'inscrit dans une politique architecturale à caractère fortement municipale opposée à l'impérialisme architectural et au style emphatique des trente années précédentes. La plupart des projets auxquels s'attèlent les architectes possèdent un caractère social, sanitaire ou médical. Beblo est le principal maître d'œuvre de l'architecture strasbourgeoise de la décennie 1904-1914. L'architecte n'est d'ailleurs pas insensible à l'héritage de l'architecture française, bien qu'il considère qu'il faille «épurer la merveilleuse ville » dénaturée par certaines constructions de l'époque française, ainsi le Palais Rohan, « corps étranger » dans la vieille ville ${ }^{18}$; mais il fustige également les excès des débuts de l'Annexion. Pour Beblo, Blondel reste une référence incontestable. Cependant, les bains municipaux apparaissent alors soit comme une référence aux palaces des stations balnéaires d'Allemagne du Nord ou de Hollande ${ }^{19}$, soit comme une expression du style «Alt deutsch ${ }^{20}$ » caractérisé par une certaine indifférence vis à vis du foyer Art nouveau de l'École de Nancy, et un regard insistant porté vers l'outre-Rhin. Le style volontairement thermal ${ }^{21}$ utilisé par Beblo l'affranchit des références antiquisantes et orientalisantes de ce type de programme, même s'il ne se dispense pas, sur l'un des projets, d'une frise de grecques dessinée sur l'architrave de la rotonde de réception. ${ }^{22}$

Si les piscines des bains municipaux de Strasbourg apparaissent vite comme les plus modernes du genre, celle de Colmar, édifiée en 1906, jouit d'une fortune critique moindre.

\section{La piscine de Colmar}

Dans les premières années du vingtième siècle, la ville de Colmar se préoccupe avant tout d'édifier des bâtiments à vocation éducative, sanitaire, sociale ou sportive: ainsi le bâtiment de la soupe populaire (actuelles Archives municipales) et les bains municipaux. À la fin des années 1880, Jean-Baptiste Krempp ouvre un bain froid en aménageant un bassin sous le barrage de l'Ill. L'entreprise ferme en 1903 et, aussitôt, l'administration militaire allemande érige près du bassin Krempp une piscine pour son propre usage, utilisée par les Sports réunis de Colmar jusque dans les années $1960^{23}$.

20 La construction d'une piscine municipale, symbole d'une ville saine et moderne ${ }^{24}$, est, comme à Strasbourg et Mulhouse, pressentie dès la fin du XIX siècle. L'idée d'un lieu où s'adonner à la natation et aux bains est aussi évoquée en 1896 dans un opuscule traitant du projet d'un établissement de bains populaires (Volksbadeanstalt) publié par l'ancien directeur de l'usine à gaz et de l'adduction d'eau de Colmar, M. G. Kern ${ }^{25}$. À partir de cette date, l'idée d'une piscine moderne ne quitte plus les services municipaux. Un projet est élaboré par l'entreprise spécialisée Göhmann \& Einhorn ${ }^{26}$ établie à Dortmund mais le budget municipal n'en permet pas l'investissement. Une réunion est ensuite organisée par le maire Riegert le 6 novembre 1899 dans le foyer du théâtre. La tentative de déléguer la maitrise d'ouvrage à une initiative privée étant demeurée lettre morte, la municipalité décide d'entreprendre la construction en régie directe. Les ingénieurs Hamann et Thomé de l'entreprise Schaffstaedt conçoivent un projet, examiné, à la demande de l'administration municipale, par des ingénieurs berlinois - dont l'inspecteur des bâtiments Matzdorff, relativement sceptique. Le projet de l'architecte Haman, critiqué en raison de son esthétique ("architektonische Ausbildung») est abandonné par la municipalité qui lui préfère le projet néo-baroque de son propre service, sous la direction de Bertsch, architecte municipal. Le projet présenté par ce dernier est adopté le 28 juillet 1903. Le coût estimé est de 330000 marks pour le gros œuvre et de 63 333,12 marks pour 
le second œuvre. Ce projet initial prévoyait deux bassins (hommes/femmes) placées symétriquement par rapport à un axe central ; seule l'une des deux piscines fut réalisée, ce qui explique la dissymétrie du bâtiment (ill. 3).

\section{3 : Vue d'ensemble des Bains municipaux de Colmar}

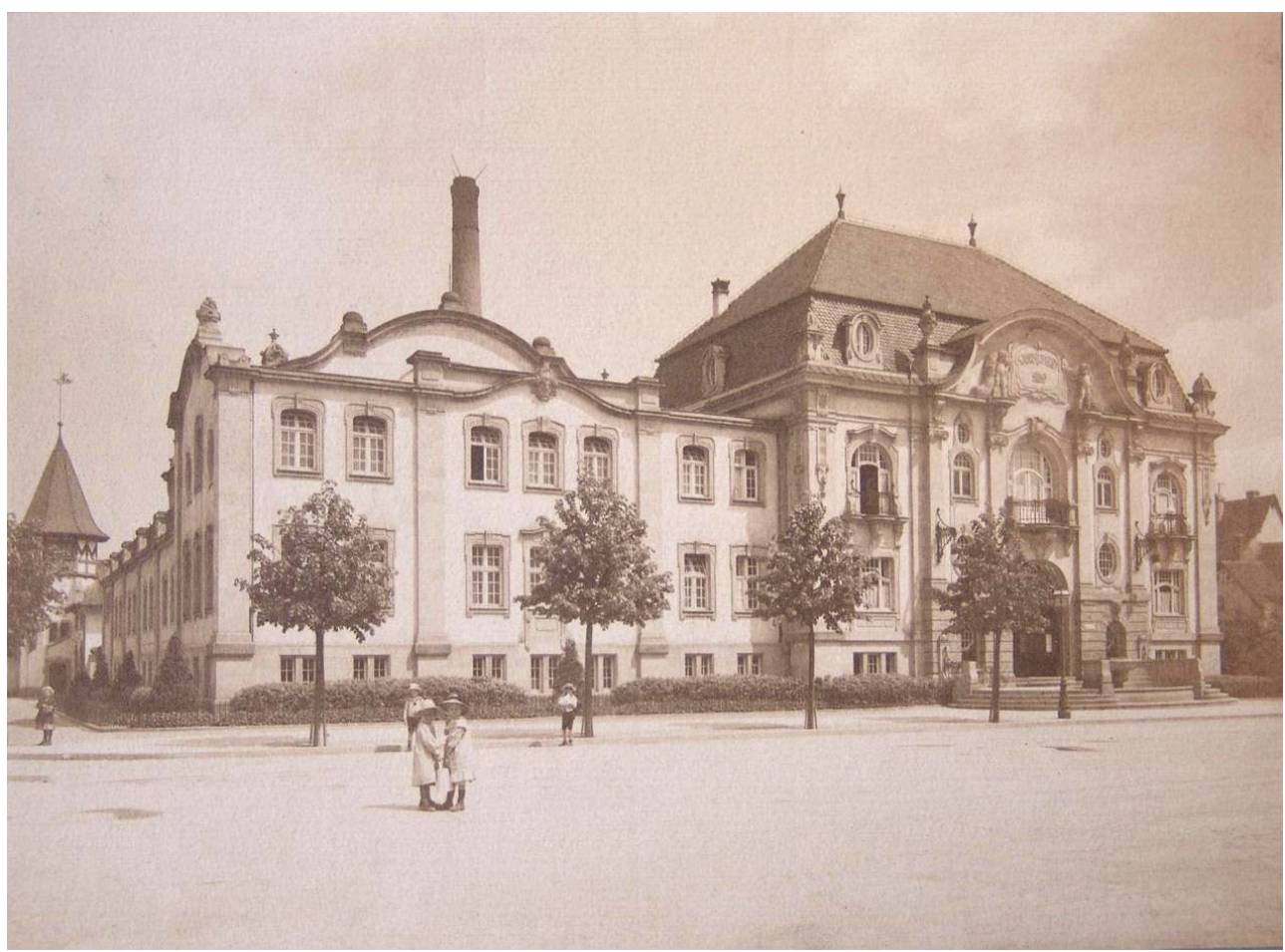

S.d.

Stadtarchiv Colmar, Archives municipales de Colmar.

Contrairement à Mulhouse et Strasbourg, où les établissements de bains sont bâtis en périphérie directe du noyau urbain primitif, les bains de Colmar s'inscrivent au sein du centre historique, face au couvent des dominicaines d'Unterlinden, sur le lieu-dit «Ackerhof ». La façade néo-baroque ${ }^{27}$, avec avant-corps et fronton curviligne, est placée en retrait de 6 mètres sur la rue Unterlinden et ménage un espace de jardinets sur rue de part et d'autre d'un escalier avec fontaine centrale semi-circulaire.

La piscine des hommes (ill. 4), en l'absence de la construction de l'aile réservée aux dames, est finalement utilisée alternativement par les deux sexes. 


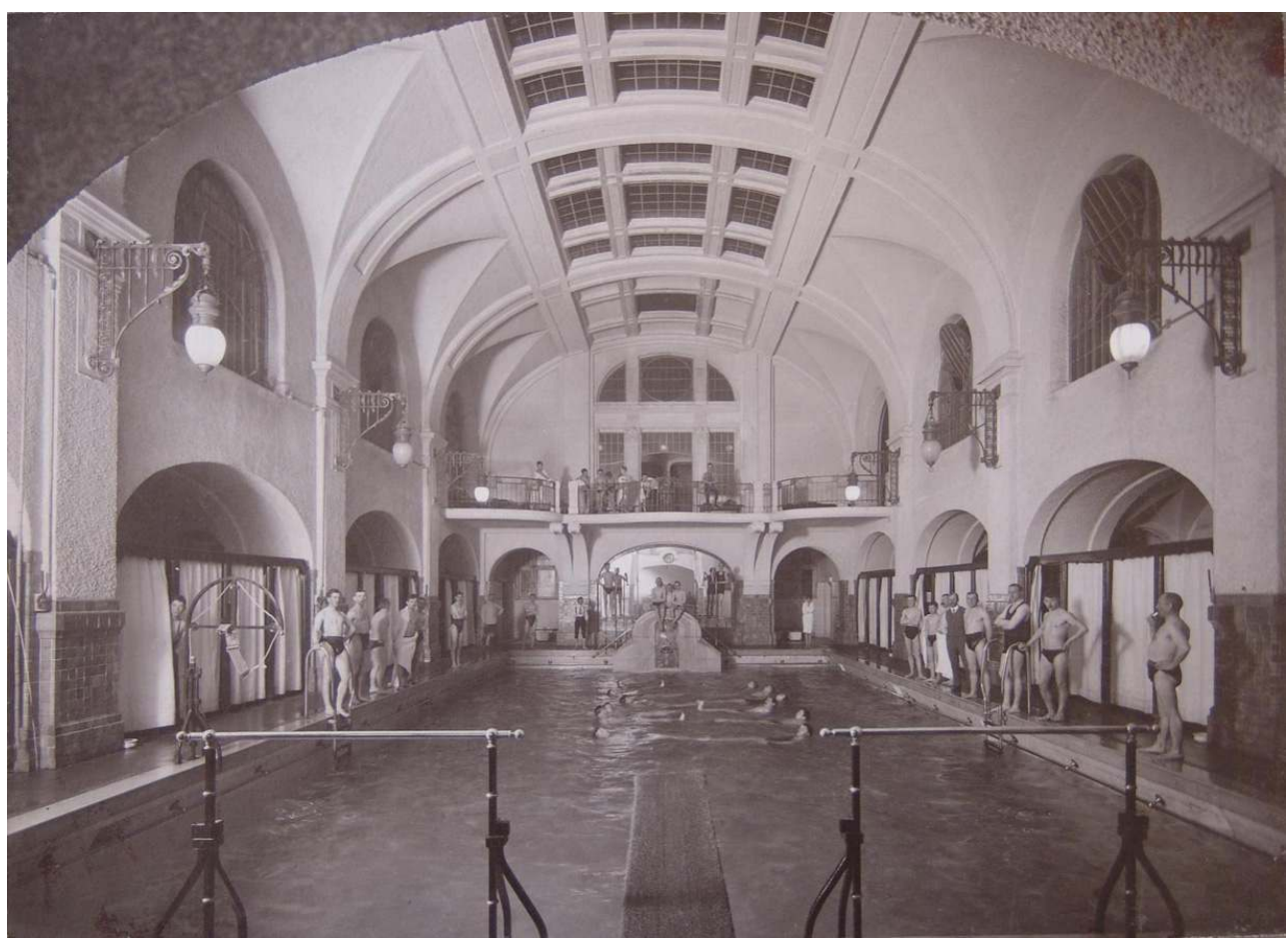

S.d., inscrit au verso $n^{\circ} 1244$

Stadtarchiv Colmar, Abteilung X, Nummer 6, Archives municipales de Colmar

Constituant initialement l'aide ouest, elle adopte le principe du plan basilical fermé par une abside semi-circulaire au nord, clos par une haute fontaine en marbre figurant la face d'un poisson barbu anthropomorphe d'où jaillit l'eau. L'élévation intérieure est à trois niveaux. Moins convenue que la façade néo-baroque, pastiche directement inspiré des palais du XVIII ${ }^{\mathrm{e}}$ siècle allemand, la piscine adapte dans son élévation extérieure le vocabulaire plus traditionnel de l'éclectisme allemand de la fin du XIX ${ }^{\mathrm{e}}$ siècle.

Elle est couverte d'un plafond cintré d'une portée de 12 mètres doté en son centre d'une vaste verrière à vitraux colorés dont les nervures de béton armé ne sont pas revêtues d'un berceau en plein-cintre ou en anse de panier, comme à Strasbourg et Mulhouse. Une tribune à l'extrémité nord forme un belvédère, et une rangée de cabines de déshabillage placées entre les arcades flanque le niveau inférieur du bassin éclairé par de grandes appliques en ferronnerie. Les lunettes en arcs brisés ouvertes sur des baies en plein-cintre confèrent à l'élévation intérieure une référence au gothique mais s'apparentent également à la piscine de Prenzlauer Berg à Berlin édifiée en 1900, en style néo-médiéval ${ }^{28}$, tandis que les appliques lumineuses de ferronnerie disposées sur chaque travée dérivent de la piscine des Müller'sches Volksbad de Munich édifiée en 1901 par Carl Hocheder.

La piscine de Colmar s'adresse à une population moins nombreuse qu'à Strasbourg ou à Mulhouse. Si elle ne constitue pas un modèle, elle s'affranchit, grâce à son budget et son échelle limités, des artifices qui monumentalisent les piscines de Strasbourg et Mulhouse, notamment les doubles galeries de cabines et la modénature néo-baroque. Ouverte en 1906, la piscine de Colmar synthétise les enjeux de la construction d'une piscine moderne et devance en cela les réalisations strasbourgeoises et mulhousiennes, c'est-à-dire la mise 
en œuvre du béton armé, un couvrement en berceau avec verrière ou non et des cabines en batterie disposées autour du bassin.

\section{La piscine de Mulhouse}

C'est Maurice Baumeister, formé aux Écoles polytechniques de Zurich et de Stuttgart, administrateur des travaux municipaux de 1911 à $1953^{29}$, qui est chargé de la conception des bains municipaux de Mulhouse, assisté de Josef Müller. Ils constituent l'œuvre principale de Baumeister et lui valent une médaille d'or et un diplôme d'honneur à l'exposition internationale de Barcelone ${ }^{30}$.

En 1911, précisément, alors que la ville est confrontée à un fort accroissement de sa population (50 790 habitants en 1900 ; 197022 habitants en 1924), elle approuve la construction d'un établissement de bains avec piscines de natation destiné à pallier la disparition progressive des bains ouvriers et des bains de rivière. Après une interruption de chantier durant la première guerre mondiale, l'établissement est inauguré en 1925. Il est alors lui aussi considéré comme le plus moderne de France, proposant un grand nombre de prestations de qualité, allant des bassins de natation au simple bain en baignoire, en passant par les bains romains ou les « douches à effet ».

La façade néo-classique d'inspiration allemande s'étend le long de la rue Curie sur près d'une centaine de mètres de long de part et d'autre d'un avant-corps central muni d'un portique à quatre colonnes d'ordre ionique et précédé d'un large degré. La façade sur rue abrite de part et d'autre du vestibule les cabines avec douches ou baignoires, réservées, au nord pour les hommes, au sud pour les femmes. Comme à Strasbourg, les bâtiments sur cour dont les combles et les toitures surpassent la façade principale, abritent les deux piscines réservées aux femmes et aux hommes (ill. 5), dont les espaces sont disposés perpendiculairement. 
III. 5 : Bains Municipaux de Mulhouse : bassin des hommes

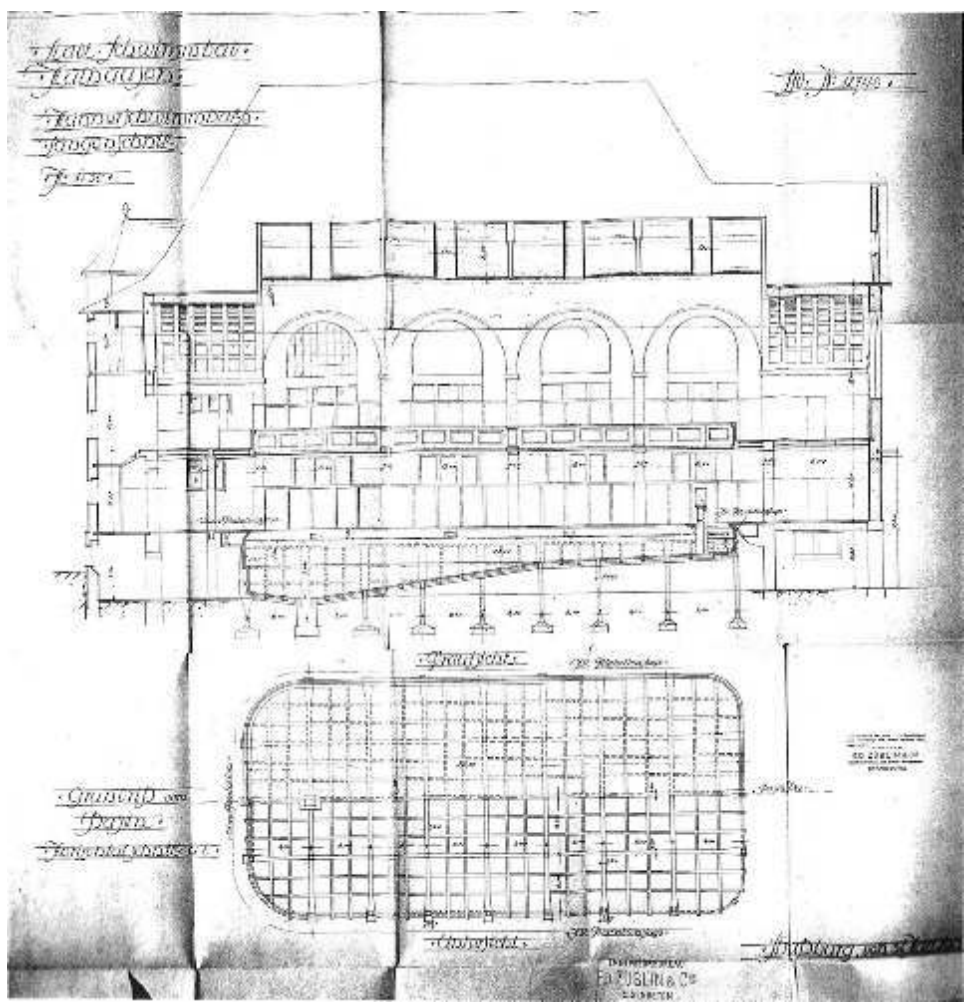

Plan du bassin ; coupe longitudinale, au 1/50e. Tirage héliogr. \& crayons de couleurs sur papier ; $103 x$ $100 \mathrm{~cm}$; Ed. Zublin \& Cie Ingenieurbureau \& Beton-Bau Gesch äft. Strassnurg. Den 31. März 1913

Archives municipales de Mulhouse, cliché service régional de l'inventaire du patrimoine culturel région Alsace, Menninger, 1984

Le corps central du bâtiment, comme à Strasbourg et Colmar, renferme les bains thermaux ou médicinaux, selon une organisation propre aux bains romains. Relié au nord, un petit bâtiment muni de grandes baies rectangulaires et d'une cheminée circulaire en brique hors oeuvre, abrite la chaudière à charbon à double entrée conçue par la Société alsacienne de constructions mécaniques, qui alimente les piscines en eau chaude.

L'accès se fait par un corridor sans vestibule. L'ordonnance de l'élévation conçue par Baumeister et Müller reprend le principe des voûtes en berceau à lunettes reposant sur des piles soutenant une galerie supérieure garnie de cabines de déshabillage (ill. 6). 


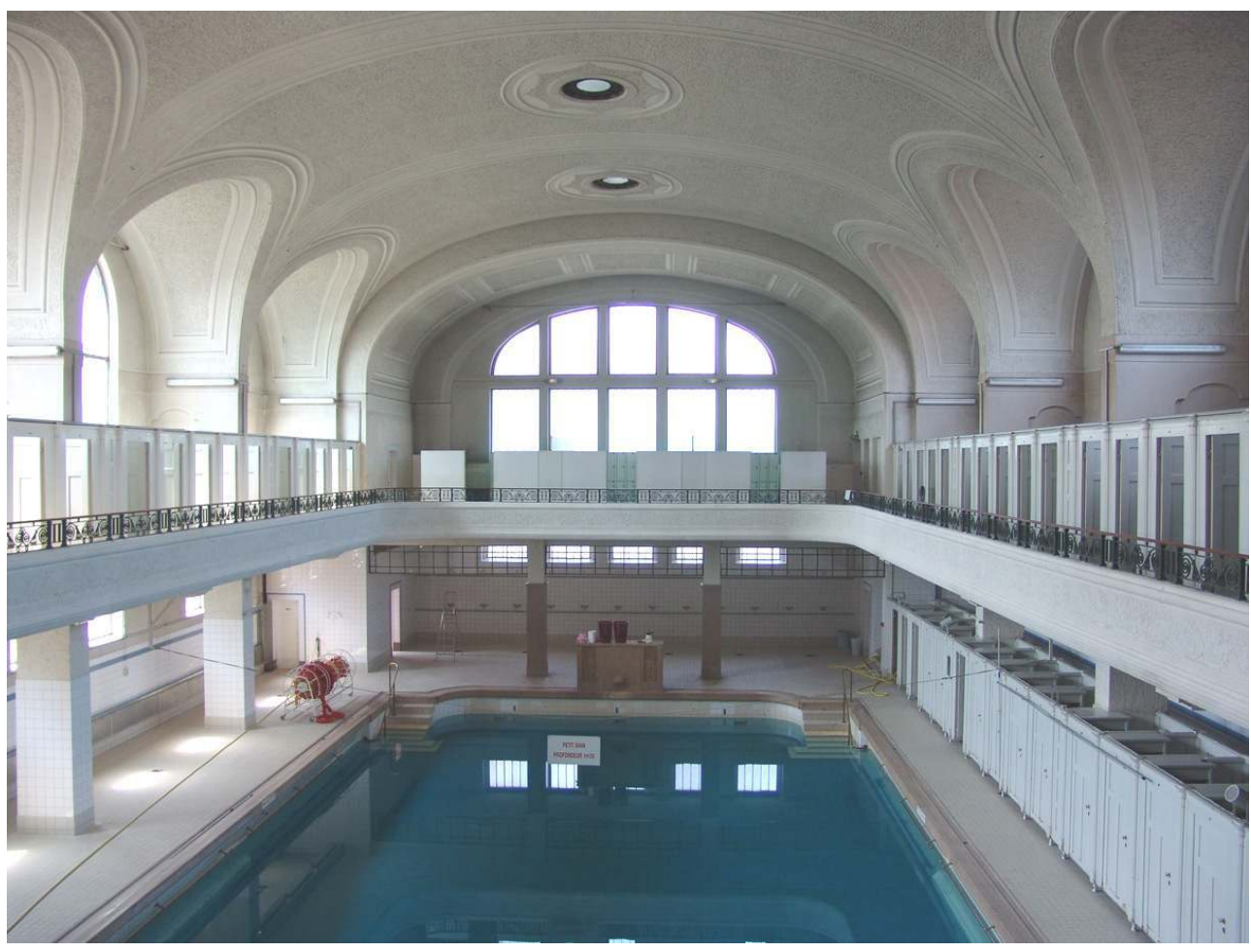

Cl. CRMH Alsace, Triboux 06487

30 Les voûtes à double paroi reçoivent une mouluraton imitant des faux caissons pour la piscine des femmes, et une mouluration soulignant simplement les travées pour la piscine des hommes, dont le bassin, d'une dimension de 12,50 m. de large pour $25 \mathrm{~m}$. de long est plus grand que celui des dames, long de $17 \mathrm{~m}$. Des oculi placés au centre des voûtes dissimulent derrière des grilles soit un éclairage électrique, soit une communication avec un lanternon en toiture, permettant ainsi un éclairage zénithal. Un niveau de tribune ferme l'extrémité de chaque piscine devant une vaste fenêtre thermale assurant un éclairage naturel. Enfin, les bassins sont arrondis à l'extrémité opposée au côté portant la fontaine.

31 Des vitraux à décor de verres colorés et à décor végétal avec inclusion de cabochons de verre réalisés en 1925 par Joseph Ehrismann garnissaient les baies des deux piscines qui ont conservé leur robinetterie en laiton, leurs pédiluves et leur réseau d'adduction d'eau.

Si le parti stylistique de la façade reprend des modèles néo-classiques allemands, les piscines reçoivent un décor tendant vers l'Art déco de façon assez marquée, notamment avec les horloges et les garde-corps de ferronnerie des galeries supérieures. Les parois des bassins sont, comme à Strasbourg et Colmar, revêtues de céramiques blanches par les établissements Zublin.

Les piscines alsaciennes conçues dès la charnière des $\mathrm{XIX}^{\mathrm{e}}$ et $\mathrm{XX}^{\mathrm{e}}$ siècles témoignent de l'intérêt des municipalités pour ce type de programme assurant tout à la fois un service d'hygiène à la population (bains) et un équipement sportif (piscine), sans que la natation comme discipline sportive soit à l'origine de ces commandes dispendieuses. Érigées durant l'Annexion avec des architectes formés outre-Rhin, les édifices se réfèrent aux programmes de piscines qui se multiplient en Allemagne à partir de 1900. Les bains de 
Strasbourg tentent de donner la réplique à ceux de Baden-Baden par Carl von Dernfeld dès $1877^{31}$, mais les modèles persistants mêlant le Jugendstil au néo-baroque sont les piscines pour hommes et femmes du Müller'sches Volksbad de Munich construites en 1901 par Carl Hocheder ${ }^{32}$. D'un point de vue technique, l'utilisation du béton armé selon la mise en œuvre établie par Zublin fait de ces édifices des bâtiments particulièrement novateurs. Enfin, d'un point de vue social et politique, ces piscines non mixtes révèlent l'affirmation du pouvoir municipal en matière d'hygiène publique. La nécessité pour ces architectes de concevoir des ensembles intégrés où les bâtiments abritant les piscines sont élevés hors œuvre, en quête de lumière naturelle, a constitué une contrainte non négligeable.

\section{NOTES}

1. Roland Oberlé, La Vie quotidienne en Alsace au temps de la Renaissance, Strasbourg, Éditions Oberlin, 1983, p. 119.

2. Catherine Grodecki, Archives du Bas-Rhin, guide des sources de l'histoire de l'art et de l'architecture en Alsace, XI XVIII siècles, Strasbourg, Presses universitaires de Strasbourg, 1996, p. 185-186.

3. Zublin et $\mathrm{C}^{\mathrm{ie}}$, Ingénieurs, entreprise de constructions en béton et béton armé et de travaux publics, Strasbourg, catalogue V: Architecture, Paris, impr. Thevoz, s.d. [1923 ?], bibliothèque nationale universitaire de Strasbourg.

4. Ibid., p. 18.

5. Arch. mun. de Mulhouse, M II a 9, Zublin \& $\mathrm{C}^{\mathrm{ie}}$, Mannerschwimmhalle, Coupe transversale sur le bassin des hommes, Strassburg, 31 mars 1913.

6. Arch. mun. de Mulhouse, M II a 15, Ed. Zublin \& C ${ }^{\text {ie }}$ Ingenieurbureau Eisenbeton, Coupe transversale et plan du bassin des hommes, 31 mars 1913.

7. Zublin \& $\mathrm{C}^{\mathrm{ie}}$, Ingénieurs, entreprise de constructions en béton..., op.cit., p. 18.

8. Dominique Toursel-Harster, Dossier de protection au titre des monuments historiques, conservation régionale des monuments historiques, DRAC Alsace, 1999.

9. Bauen auf Tradition, Fritz Beblo 1872-1947, Stadtbaurat in Strassburg und München, Austellung im Stadtarchiv München, 25 sept 1991 - 9 nov. 1991, p. 27.

10. Ibid., p.29.

11. Shelley Hornstein-Rabinovitch, Tendances d'architecture Art nouveau à Strasbourg, thèse de doctorat, Strasbourg, université des sciences humaines, institut d'histoire de l'art, octobre 1981.

12. Klaus Nohlen, Construire une capitale. Strasbourg impérial de 1870 à 1918 - Les bâtiments officiels de la Place impériale, Collection « Recherches et documents », t. 56, publications de la société savante d'Alsace, 1997, p. 189 et suiv.

13. Georges Foessel, Jean-Pierre Klein, Strasbourg, panorama monumental et architectural des origines à 1914, Barcelone, Mémoire d'Alsace G4J, 2003, p. 325.

14. Arch. mun. de Strasbourg, 947 W 68, piscine de la ville de Strasbourg, Plan du rez-de-chaussée, signé et daté : Beblo, déc. 1905. 
15. Arch. mun. de Strasbourg, 709 W 153, piscine de la ville de Strasbourg, Élévation de la façade principale sur le Nicolausring, signé et daté : Fettig, 1906.

16. Alexis Fassel, La Natation aux bains municipaux de Strasbourg (1920-1950), A. Rauch (dir.), Sports et loisirs en Alsace au XX $X^{e}$ siècle, Paris, centre de recherches européennes en éducation corporelle / Revue E.P.S., 1994, p. 31.

17. Ibid., p. 35.

18. Denis Durand de Bousingen, "L'architecture à Strasbourg de 1903 à 1918 : styles, écoles et hommes ", Annuaire de la Société des Amis du Vieux-Strasbourg, Strasbourg, 1985, p. 60.

19. Ibid., p. 67.

20. François Loyer, Histoire de l'architecture française de la Révolution à nos jours, Paris, Mengès / Éditions du patrimoine, 1999, p. 198.

21. Théodor Rieger, D. Durand de Bousingen, Strasbourg Architecture 1871-1918, Collection Art Alsace, Obernai, Le Verger éditeur, 1991, p. 27.

22. Arch. mun. de Strasbourg, $709 \mathrm{~W} \mathrm{153,} \mathrm{Coupe} \mathrm{longitudinale} \mathrm{sur} \mathrm{la} \mathrm{rotonde} \mathrm{de} \mathrm{réception,} \mathrm{signé} \mathrm{et}$ daté : Beblo, 1906.

23. Gabriel Braeuner, Francis Lichtlé, Dictionnaire historique de Colmar, Association pour la restauration des édifices historiques de Colmar, Colmar, 2006, p. 31.

24. Thomas Goldstein, Intégration d'un bâtiment accueillant de nouveaux bains municipaux dans le centre historique de Colmar, mémoire de TPFE, Diego Peverelli (dir.), école nationale supérieure d'architecture de Strasbourg, septembre 1999, p. 6.

25. Philippe Heinitz, Les Relations entre architecture et urbanisme dans le Reichsland, 1870-1914, éléments pour une histoire urbaine de la ville de Colmar, mémoire de D.E.A. sous la dir. de Pier Giorgio Gerosa, 9 oct. 2003, 2e éd. 2005, p. 160.

26. Arch. mun. de Colmar, 786, Errichtung eines volksbads.

27. Direction de l'architecture de la ville de Colmar, janvier 1984, non coté, Bains municipaux : façades sud et ouest au 1/100e.

28. Kristin Feireiss, Badetempel: Volksbader aus Gründerzeit und Jugendstil, Berlin, Ernst \& Sohn, 1993, p. 46.

29. Charles Seither, « Maurice Baumeister 1880-1954 », Bulletin du musée historique de Mulhouse, t. LXIV, 1956, p. 155.

30. Ibid., p. 156.

31. Kristin Feireiss, op. cit., p. 26.

32. Ibid., p. 50.

\section{RÉSUMÉS}

Les premières années du $\mathrm{XX}^{\mathrm{e}}$ siècle voient se concrétiser à Colmar, Strasbourg et Mulhouse trois importants programmes de bains municipaux avec bassins de natation et bains médicinaux dans la lignée des programmes de bains déterminés en Europe centrale et en Allemagne à la charnière des $\mathrm{XIX}^{\mathrm{e}}$ et $\mathrm{XX}^{\mathrm{e}}$ siècles. Les piscines qui $\mathrm{y}$ sont conçues dans une perspective de non-mixité participent alors d'un projet sanitaire et social global à visée hygiéniste, en cette période où l'Alsace est encore Reichsland d'Empire mais souhaite se détourner des commandes impériales, ou 
se consacrer à des chantiers à destination du peuple. Ni totalement Art nouveau ni complètement éclectiques, ces piscines mises en œuvre avec le concours du savoir-faire pionnier de l'entrepreneur en béton armé Édouard Zublin parviennent à doter les capitales alsaciennes d'équipements nautiques capables de rivaliser avec les plus récentes réalisations des grandes cités allemandes.

In the early years of the $\mathrm{XX}^{\text {th }}$ century were carried out three important municipal baths programs, in Colmar, Strasbourg and Mulhouse, with swimming pools and medicinal baths in the wake of the baths programs determined in Central Europe and Germany at the turn of the XIX ${ }^{\text {th }}$ century. The swimming pools, planned for a non mixed public, were part of an overall hygienistdesigned social and health project, at a time when Alsace as a Reichsland was still annexed to the Empire but intended to turn away from imperial commissions or to devote itself to some new constructions for its people. Neither completely Art nouveau, nor completely eclectic, these swimming pools built with the help of the leading expertise of the reinforced concrete contractor ED Zublin, enabled the Alsatian capitals to get swimming facilities that were likely to compete with the most recent achievements of the big German cities.

In den ersten Jahren des 20.Jahrhunderts kommen drei bedeutsame Bauprogramme von städtischen Bädern mit Schwimmbecken und Heilbädern zustande. Sie übernehmen die Tradition der Badeeinrichtungen in Mitteleuropa und in Deutschland um die Jahrhundertwende. Die Gesamtplanung dieser Badeanstalten verfolgt sanitäre, soziale sowie hygienische Absichten. In dieser Zeit versucht das Elsass, immer noch deutsches Reichsland, sich gegen kaiserliche Aufträge zu wehren und bevorzugt selber für die Bevölkerung zu bauen. Weder ganz im Jugenstil noch völlig im eklektischen Stil werden diese Badeanstalten dank des technischen Könnens des Unternehmers Ed. Zublin aus Stahlbeton gebaut. So verfügen die elsässischen Hauptstädte über Schwimmbadausstattungen, die den Vergleich mit den modernsten Anstalten der deutschen Gro ßstädte nicht zu scheuen brauchen.

INDEX

Index chronologique : époque contemporaine, XIXe siècle, XXe siècle

Mots-clés : piscine

Keywords : swimming pool

Schlüsselwörter : Schwimmbad

\section{AUTEUR}

\section{PATRICE TRIBOUX}

Patrice Triboux, né en 1970, après un premier cycle à l'École nationale supérieure d'architecture de Marseille-Luminy, a suivi des études d'histoire de l'art et d'archéologie à l'université de Provence. Son mémoire de maîtrise en sciences et techniques de l'archéologie et du patrimoine méditerranéens a porté sur l'étude du parc archéologique et des antiques de Glanum sous la direction de Brigitte Sabattini. Conférencier agréé par le centre des monuments nationaux en 1995, puis conférencier pour la direction des musées de Marseille et conférencier pour la réunion des musées nationaux, il est en poste au département des estampes et de la photographie de la Bibliothèque nationale de France entre 1998 et 2000. Il œuvre ensuite au musée national Picasso comme chargé d'études où il publie des articles sous la direction de Dominique Dupuis-Labbé et est commissaire de l'exposition « Picasso et la Grèce » à la Fondation Goulandris en 2004. Il est 
chargé de la protection des monuments historiques de la région Alsace depuis 2006. Adresse électronique : patrice.triboux@culture.gouv.fr 